\title{
СОВЕРШЕНСТВОВАНИЕ ПРАКТИКИ УПРАВЛЕНИЯ РИСКАМИ ПРИ РАЗРАБОТКЕ ПРОДУКЦИИ НА ПРЕДПРИЯТИЯХ КОСМИЧЕСКОЙ ОТРАСЛИ
}

\author{
(c) 2021 Афанасьева Елизавета Александровна \\ аспирант кафедры экономического управления организацией \\ Оренбургский государственный университет, Россия, Оренбург \\ E-mail: afanasjeva.elisabeta@yandex.ru
}

Разработка космической продукции является важным видом деятельности для предприятий космической отрасли, перед которыми стоит задача не только постоянно производить новую продукцию, но и сокращать время разработки, снижать затраты, повышать качество продукции. Западные космические компании успешно ответили на этот вызов, внедрив практику бережливого производства, сосредоточившись на критической ценности и отслеживая нерациональное использование ресурсов. Однако управление рисками все еще плохо интегрировано в процесс разработки продукции, в том числе в России, хотя оно сильно влияет на результативность процесса разработки продукции [1]. То есть, имеется потребность в совершенствовании практики управления рисками при разработке продукции на предприятиях космической отрасли, что и является целью данного исследования. Для достижения данной цели, посредством обзора литературы, нужно осветить различные практики управления рисками, управления разработкой продукции и модели зрелости, применяемые в космической отрасли.

Объект - российские и зарубежные предприятия космической отрасли.

Предмет - управленческие отношения, возникающие в процессе управления рисками при разработке продукции на предприятиях космической отрасли.

\section{Результаты:}

1) Получено четкое представление о различных методах, используемых в настоящее время при управлении рисками и управлении разработкой продукции.

2) Инженерам и руководству предоставлен список актуальных, специфичных для космической отрасли критериев оценки результативности и зрелости процесса разработки продукции, которые могут быть ими использованы в качестве основы для будущих процессов принятия решений. Результаты имеют прикладную направленность и могут быть использованы в управлении рисками при разработке продукции на предприятиях ракетно-космической отрасли России.

Ключевой вклад. Установлены конкретные характеристики, которые могут быть использованы в качестве эталонной практики управления рисками при разработке космической продукции.

Источниками информации стали российские и зарубежные научные публикации.

Методы исследования: анализ, синтез, классификация, сравнение, абстрагирование, обобщение, моделирование.

Ключевые слова: разработка продукции, управление рисками, зрелость, результативность, показатели, космическая отрасль.

\section{Введение}

В связи с усилением глобальной конкуренции, технологическим прогрессом и быстро меняющимися запросами потребителей, сокращение или просто соблюдение сроков разработки продукции является стратегическим приоритетом для многих предприятий [9]. Поэтому эффективное планирование считается важным для сокращения продолжительности проекта. Так в исследовании [14] отмечено, что тщательное планирование на начальной стадии проекта напрямую влияет на его эффективность: оно уменьшает неопределенность, особенно техническую. Также в работе [9] указывается, что плохое планирование может привести к задержкам и перерасходу средств до 70\%, что можно наблюдать во многих масштабных проектах. Данные проблемы появляются, когда применяемые методы управления не принимают во внимание существование итераций процесса разработки и связанных с ними рисков, что является необходимым условием для разработки эффективной стратегии планирования, ускорения и улучшения процессов разработки продукции [9]. 
Совершенствование практики управления разработкой продукции в космической отрасли

В каждой организации есть цели, состоящие из поставленных задач, которые определяют ожидаемые результаты и направляют усилия сотрудников. Когда есть цель, тогда составляется план по достижению этой цели, то есть проект. В данной работе [13] отмечается: «Процесс ... означает работу, которая переходит от одного шага к другому. Каждый шаг может представлять собой набор запланированных задач. Таким образом, рабочий список (список экземпляров процесса, которые необходимо выполнить) может быть преобразован в ... график проекта... проект может быть вложен в процесс».

Существует множество инструментов, чтобы помочь руководителям планировать свои проекты. Наиболее распространенными методами планирования проектов являются: диаграмма Ганта; метод критического пути; диаграммы PERT. Однако эти традиционные и старые методы плохо подходят для эффективной помощи в планировании и управлении сложными процессами разработки продукции. Они не учитывают итерации", вызванной вынужденной переделкой определенных задач. Наконец, они не учитывают риски, связанные с каждой задачей и развивающиеся на протяжении всего процесса разработки. В больших системах, таких как разработка продукции в космической промышленности, взаимозависимости являются обычным явлением. Именно поэтому существуют другие методы, с использованием матричного представления [8]. Отдельные задачи или объекты процесса располагаются в квадратной матрице, где пересечение каждой строки и столбца соответствует зависимости (рисунок 1).

Затем можно проанализировать отношения между этими объектами, чтобы определить и оптимизировать ключевые структуры и процессы проекта. Кроме того, в матрице может быть представлена дополнительная работа, создаваемая каждой итерацией. Эта матрица называется матрицей структуры зависимостей, или DSM. Значения в каждой строке указывают на зависимость от других объектов. Чем выше значение, тем больше их зависимость. Диагональные записи матрицы равны нулю, поскольку компонент рассматриваемой системы не может влиять сам

\footnotetext{
* Итерация - это процедура, в которой повторение последовательности операций приводит к результатам, последовательно приближающимся к желаемому. https://www.merriam-webster.com/dictionary/iteration
}

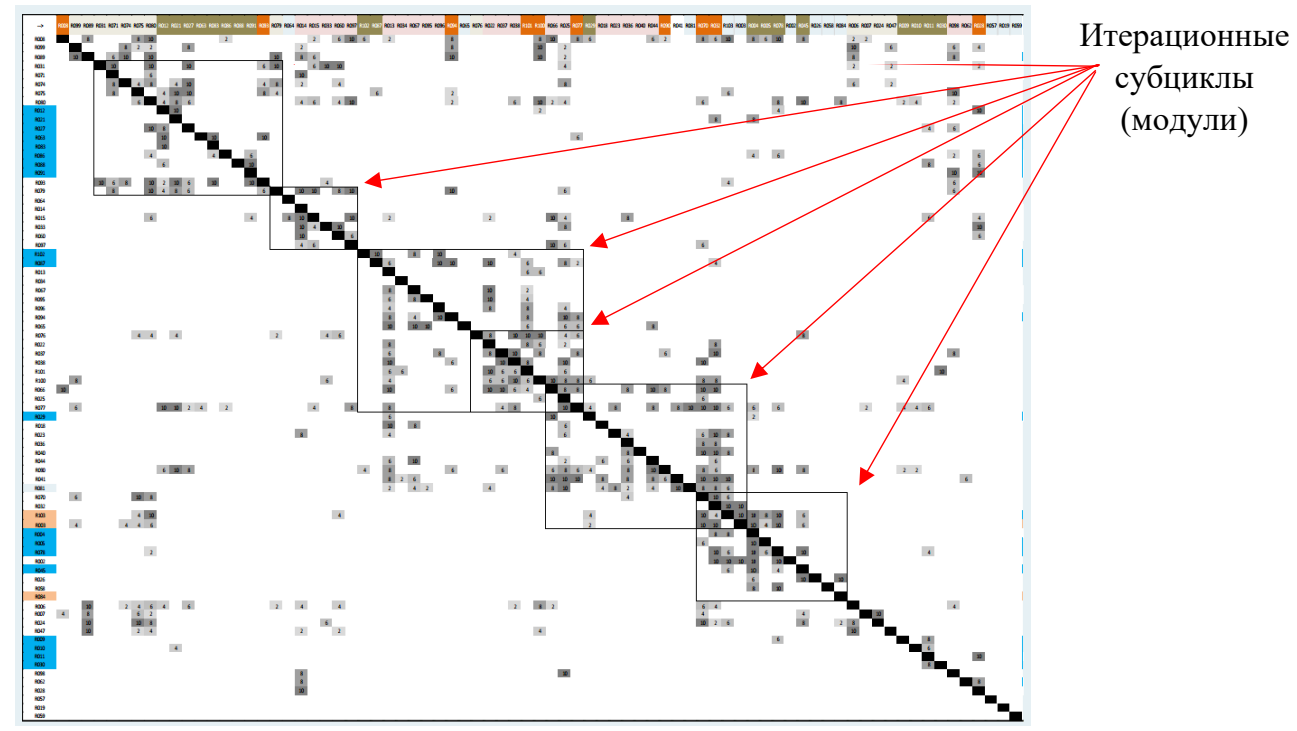

Puc. 1. DSM-матрица «риск-риск» с возможными модулями (подсистемами), определенными в предлагаемой архитектуре продукта" [8]

\footnotetext{
* Архитектура продукта - это схема, с помощью которой функции продукта распределяются между физическими компонентами.

Например, воздухонагреватель, увлажнитель воздуха, система напорных воздуховодов, охладитель воздуха, система вытяжных каналов с регенератором являются модулями архитектуры климатической камеры.

https://www.sciencedirect.com/science/article/abs/pii/0048733394007753

https://www.mas.bg.ac.rs/_media/istrazivanje/fme/vol39/4/01_kosman.pdf
} 
на себя. Для лучшего применения матричного метода процесс должен быть максимально разбит на отдельные объекты (задачи/подпроцессы).

Чтобы достигнуть нужных результатов, процесс разработки продукции должен быть хорошо спланирован, а результаты проконтролированы. Количественная оценка фактически полученных результатов процесса называется результативностью (performance) процесса. Измерение результативности процесса разработки продукции остается сложной задачей для компаний [3]. Помимо этого, при неправильном или неэффективном выполнении оно может представлять риск для организации [10]. Так, вводящие в заблуждение показатели могут привести к ошибочной оценке программы до начала ее реализации, что приводит к принятию неверных решений, которые негативно сказываются на текущем проекте. Таким образом, надежность измерительной системы имеет большое значение.

Результативность характеризуется двумя типами показателей:

1) одни относятся к самим процессами (например, время выполнения);

2) другие - к тому, что процессы создают (например, надежность выполненной работы или количество ошибок).

В статье [12] перечислены параметры для оценки результативности процесса разработки. Разработанный в статье [12] инструмент LAT (Lean-инструменты оценки) структурирован по восьми измерениям:

1) эффективность использования времени;

2) качество;

3) процесс;

4) стоимость;

5) человеческие ресурсы;

6) цепочка поставок;

7) потребитель;

8) запасы.

Для каждого измерения предлагается список собственных показателей. В результате получается 51 показатель оценки результативности. Другой список из 50 метрик разработан в работе [10], из которых в качестве наиболее полезных предлагаются:

1) удовлетворенность клиентов;

2) процент своевременной сдачи проекта;

3) удовлетворенность сотрудников;

4) разница между фактическим и запланированным временем завершения проекта;
5) точность интерпретации требований заказчика;

6) общая стоимость проекта;

7) количество и характер узких мест.

Эти данные являются результатом отраслевого опроса, основанного на небольшой выборке из 25 ответов от представителей оборонной промышленности.

Разработка космической продукции - это очень сложная работа, которая требует и одновременно генерирует огромные объемы данных и знаний. Поэтому концепция зрелости знаний [7] и зрелости процесса разработки имеет важное значение.

Зрелость - это мера способности организации к постоянному совершенствованию в определенной дисциплине (которая считается зрелой если использовалась и развивалась достаточно долго, и большинство ее первоначальных проблем были преодолены). Чем выше уровень зрелости, тем выше результаты в целом и тем меньше вероятность того, что инциденты или ошибки приведут к потерям в качестве или использовании ресурсов. Оценка зрелости может производится в отношении процессов, технологий, риска, знаний и др. [15]

В работе [7] концепция зрелости знаний рассматривается как: «средство обеспечения практической поддержки принятия решений, которое повышает осведомленность лиц, принимающих решения, о базе знаний и поддерживает межграничные дискуссии о воспринимаемой зрелости имеющихся знаний». И предлагается: «Создать меру зрелости, где состояние готовности актива знаний оценивается с помощью шкалы, насколько «пригодна к использованию» часть знаний в отношении целей». Авторы также отмечают, что: «Зрелость знаний основывается на других концепциях зрелости - уровне готовности технологий и модели зрелости возможностей (CMM), для применения этих идей в контексте знаний».

Зрелость процесса относится к степени, в которой он четко определен, управляется, измеряется и контролируется. Зрелость предполагает потенциал роста возможностей и является показателем степени согласованности действий организации в удовлетворении требований конечных потребителей. Были разработаны модели, определяющие структуру оценки зрелости. СММI («Интеграция модели зрелости возможностей») 
используется для независимого сравнительного анализа критических бизнес-процессов и технических процессов. Согласно источнику [7]: «СММI является развитием методологии СММ, используется государственными учреждениями для оценки подрядчиков программного обеспечения ... достижение достаточно высокого уровня по этой шкале является гарантией того, что компания надежна в поставках, что снижает общий риск для заказчика». Набор продуктов CMMI был разработан с целью объединения нескольких моделей развития процессов в единую систему совершенствования для использования организациями, стремящимися к комплексному улучшению процессов.

В CMMI описаны конкретные практики, указывающие на то, что необходимо для хорошей системной инженерии. СMМI v 2.0 определяет для этого 5 уровней зрелости процессов [6]:

Уровень зрелости 0 «Неполный»: ситуативный и неизвестный, работа может или не может быть завершена.

Уровень зрелости 1 «Начальный»: Процессы практически неизвестны и непредсказуемы. Фактор успеха не определен. Успех проекта остается случайным. Работа выполняется, но часто с задержками и превышением бюджета.

Уровень зрелости 2 «Регулируемый»: основные процессы управления проектом определены, проекты планируются, выполняются, измеряются и контролируются.

Уровень зрелости 3 «Определенный»: Про- цессы проекта четко идентифицированы и определены. Все заинтересованные стороны проекта имеют четкое представление о них. Общеорганизационные стандарты обеспечивают руководство по проектам, программам и портфелям проектов.

Уровень зрелости 4 «Количественно-управляемый»: Процессы измеряются и контролируются. Организация управляется данными и имеет количественные цели по улучшению работы, которые предсказуемы и соответствуют потребностям внутренних и внешних заинтересованных сторон.

Уровень зрелости 5 «Оптимизирующий»: Процессы оптимизированные и стабильные. Организация ориентирована на постоянное совершенствование и построена так, чтобы реагировать на возможности и изменения, стабильность организации обеспечивает платформу для гибкости и инноваций.

Если СMМI позволяет охарактеризовать уровень зрелости процесса, то шкала TRL характеризует уровни технологической готовности. Согласно источнику [7]: «TRL - это подход, разработанный NASA для оценки состояния готовности к полетам путем оценки зрелости технологий (т.е. компонентов, систем и т.д.). TRL используется как производителями, так и правительственными агентствами при приобретении технологий. Производители должны продемонстрировать определенную готовность, или доказанные возможности, своих технологий и

Таблица 1. Стадии зрелости процесса и TRL в Boeing [5]

\begin{tabular}{|c|c|c|}
\hline Стадии зрелости & TRL & Содержание уровня TRL \\
\hline \multirow{3}{*}{ Открытие } & 1 & Основные принципы, соблюдаемые и сообщаемые \\
\hline & 2 & Сформулированная концепция и/или применение технологии \\
\hline & 3 & $\begin{array}{l}\text { Аналитическая и экспериментальная критическая функция и/или характе- } \\
\text { ристика пробной концепции }\end{array}$ \\
\hline \multirow{2}{*}{ Осуществимость } & 4 & Проверка компонентов и/или макетов в лабораторных условиях \\
\hline & 5 & Проверка компонентов и/или макетов в соответствующей среде \\
\hline Практичность & 6 & $\begin{array}{l}\text { Демонстрация модели системы/подсистемы или прототипа в соответству- } \\
\text { ющей среде (наземной или космической) }\end{array}$ \\
\hline \multirow{3}{*}{ Применимость } & 7 & Демонстрация прототипа системы в космической среде \\
\hline & 8 & $\begin{array}{l}\text { Действующая система завершена и получила летную квалификацию в ре- } \\
\text { зультате испытаний и демонстраций (наземных или космических) }\end{array}$ \\
\hline & 9 & Действующая система, проверенная в ходе успешных полетов \\
\hline
\end{tabular}


систем, чтобы получить контракт». TRL основан на шкале 1 до 9, где 9 соответствует наиболее зрелой технологии (таблица 1 ).

В таблице показана характеристика зрелости процесса по отношению к уровням шкалы технологической зрелости TRL. TRL упрощает процессы в космической промышленности, предоставляя общий язык для оценки зрелости технологий и технологических рисков. Модели TRL и CMMI - это признанные подходы, широко используемые в промышленности США.

Совершенствование практики управления рисками на предприятиях космической отрасли

Согласно «Руководству по управлению рисками, проблемами и возможностями для программ оборонных закупок Министерства обороны США» 2017: «Все оборонные программы сталкиваются с рисками и проблемами и должны предвидеть и решать их на постоянной основе. Управление рисками и управление проблемами тесно связаны и используют схожие процессы».

ГОСТ Р ИСО 31000-2019 определяет риск как «следствие влияния неопределенности на достижение поставленных целей», а управление рисками как скоординированные действия по руководству и управлению организацией с учетом риска. В Руководстве Министерства обороны США процесс управления рисками включает в себя планирование методов управления рисками, выявление рисков и их анализ, для разработки вариантов смягчения последствий. Затем обеспечивается мониторинг рисков, чтобы определить, как они меняются в процессе работы. Практически все этапы данного процесса содержатся в процессе управления рисками ГОСТ Р ИСО 31000-2019, применимом к любому процессу, в данном стандарте идентификация и анализ рисков объединены в этап «Оценка риска». На рисунке 2 иллюстрируется этот непрерывный процесс управления рисками (Continuous risk management, CRM), определяемый НACA («Руководство НАСА по управлению рисками» 2011) и Министерством обороны США.

Процессы разработки инженерных систем можно рассматривать как имеющие цикли-

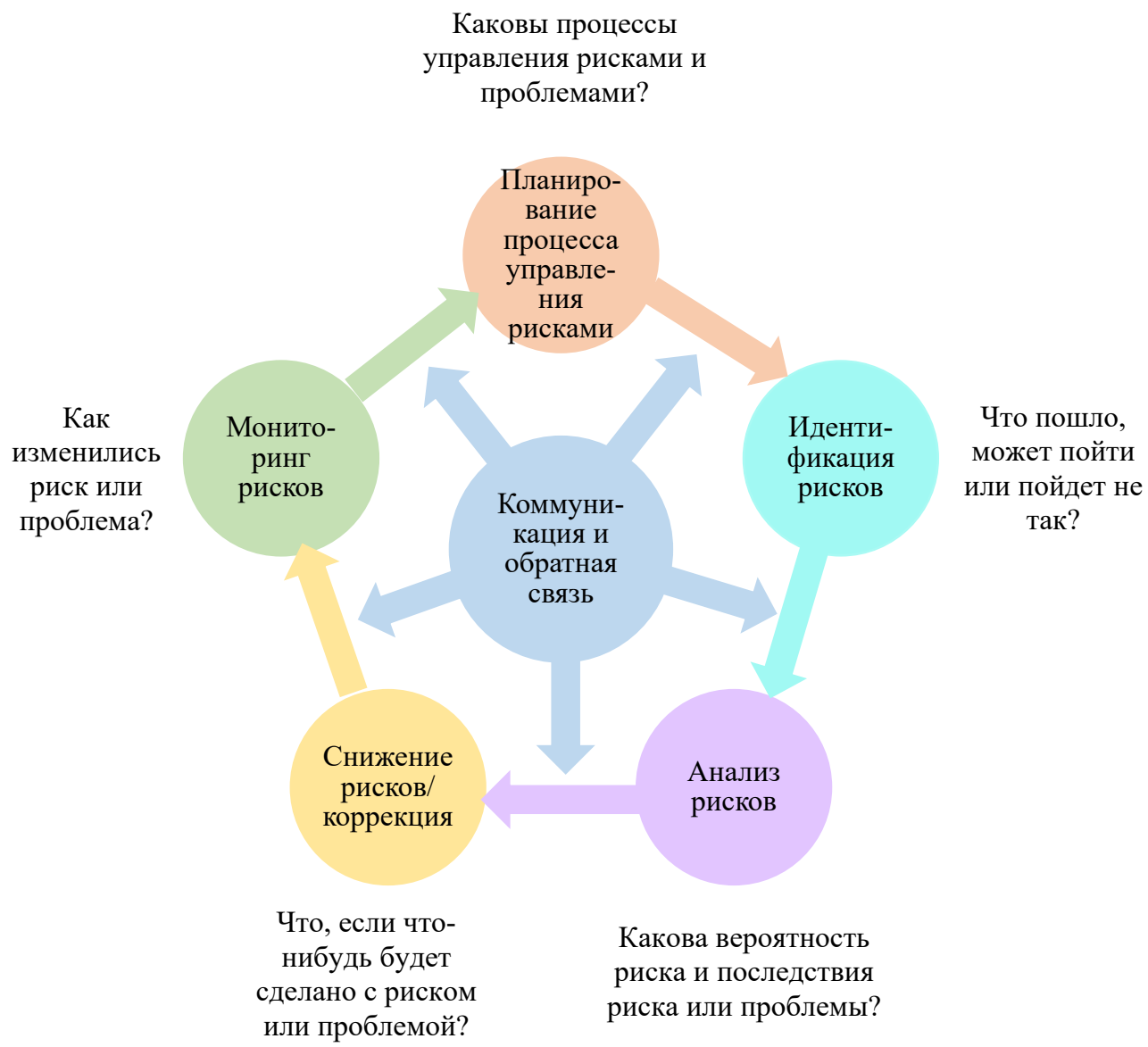

Рис. 2. Непрерывный процесс управления рисками и проблемами, определяемый Министерством обороны США 
ческое (Руководство НАСА) и итеративное [9] развитие. Поэтому оценка риска может применяться на всех этапах этого цикла и, как правило, используется многократно и с разной степенью детализации.

Чтобы помочь в принятии решений на каждом этапе, так как на разных этапах разные потребности и разные методы принятия решений. На этапе проектирования и определения можно использовать оценку рисков, чтобы решить, делать ли инвестиции. Так же, когда доступно несколько вариантов, можно оценить альтернативные концепции и решить, какой из них лучший. Поэтому оценку риска следует использовать в разработке процедур для стандартных условий и требований для каждого этапа цикла разработки. Все это должно помочь сформировать обоснованное суждение, позволяющее максимизировать эффективность распределения ограниченных ресурсов.

NASA и Министерство обороны США, определяют, что цель этапа «Идентификация» это: «фиксация опасений заинтересованных сторон относительно достижения требований к результативности. Эти опасения фиксируются в виде отдельных рисков в базе данных рисков. Каждый отдельный риск формулируется в виде заявления о риске». В Министерстве обороны США предлагаю так описывать риск: «Формат «если - то» представляет возможное событие или условие риска («если») и потенциальный результат или последствие (последствия) («тогда»)».

Для стандартизации оценки риска в ГОСТ Р 58771-2019 подробно описаны технологии применимые к любому процессу. Рисунок и таблица, взятые из приложения А этого стандарта, обобщают и позволяют сравнить технологии, доступные для оценки рисков проекта или задачи. Рисунок классифицирует технологии (сценарный анализ, анализ дерева решений, метод «галстук бабочка» и т.д.). в соответствии с их применимостью к различным этапам процесса управления рисками. Таблица идентифицирует эти технологии в соответствии со следующими признакам: степень применения усилий; способность технологии обеспечивать количественный результат; необходимость информации/данных; опыт специалиста, применяющего технологию. Каждая технология оценивается по трехбалльной шкале (высокий, средний или низкий).

Фактически, оценка риска сводится к определению сценария неопределенности, оценке частоты и причин возникновения риска и, наконец, оценке влияния его последствий. Эта оценка основана на изучении всей соответствующей информации, на основе которой будут приниматься управленческие решения. После оценки рисков на этапе «Снижение рисков», согласно Руководству Министерства обороны США, нужно разработать стратегию управления рисками, оценив четыре варианта снижения рисков: избегание, снижение, передача или принятие риска. Избегание характеризуется выбором такого пути развития, который больше не будет подвержен влиянию риска. Это также может означать принятие мер, полностью ограничивающих риск. Этот случай предусматривает замену опасной деятельности другой деятельностью, но сначала необходимо убедиться, что замена или избегание не приведет к увеличению риска или неопределенности. Передача риска отражает способы передачи ответственности и последствий риска третьей стороне. Принятие остается вариантом управления, который заключается в принятии обоснованного решения ничего не предпринимать в отношении риска и принятии полной ответственности как за решение, так и за последствия, которые могут возникнуть.

Центральное место в процессе управления рисками занимает коммуникация и обратная связь различных внутренних подразделений компании. Фактически, цель состоит в том, чтобы постоянно синтезировать внутренние и внешние риски, чтобы ими можно было эффективно управлять. Именно такой синтез информации от различных участников проекта будет способствовать его успеху и хорошим показателям эффективности компании. Министерство обороны США резюмирует эту потребность руководителей проектов в реализации эффективной стратегии управления рисками: «ель заключается как в снижении рисков, так и в создании возможностей для развития технологий, которые могут оказать положительное влияние на достижение целей результативности, а также пороговых значений». В ГОСТ Р ИСО 310002019 указано, что получение обратной связи от участников процесса необходимо для ее учета при принятии решений и осуществлении других видов деятельности.

Проектирование и разработка инженерных систем включает в себя множество решений, которые требуют баланса между различными техническими и коммерческими соображениями. 
Все это должно быть совместимо с ожиданиями всех заинтересованных сторон. Чтобы помочь принимать эти решения, необходим формальный, логичный и последовательный процесс. Последний также должен иметь возможность развертывания в течение всего процесса разработки продукта. Принятие решений в таких условиях теперь известно как «принятие решений с учетом рисков» (RIDM). Это понятие впервые появилось в «Руководстве НАСА по управлению рисками» 2011. RIDM предназначен для помощи группе, состоящей из нескольких заинтересованных сторон, каждая из которых может иметь свои приоритеты в подходе к решению проблемы, свои цели со множеством последствий и неопределенностей. Хотя методы RIDM могут быть применены к широкому кругу проблем принятия решений, основное внимание уделяется тем, которые возникают на ранних стадиях проектирования при разработке инженерных систем. Чтобы преодолеть отсутствие контроля в реальном времени и способствовать упреждающему управлению рисками, появился метод, дополняющий RIDM: непрерывное управление рисками (CRM). Эти два аспекта работают вместе для обеспечения эффективного управления рисками в программах и проектах, которые НАCA разрабатывает, развивает и реализует.

Таким образом, выделяются два вида инструментов:

1) методы, позволяющие принимать решения на начальных этапах разработки продукта;

2) инструменты, позволяющие в реальном времени отслеживать изменения или появление новых рисков.

Заметим, что время является ключевой переменной при принятии решений. На предварительных этапах обычно требуется больше времени для выбора основных направлений проекта. Когда руководитель переходит к следующей стадии проекта и сталкивается с ранее не выявленными рисками, от которых нельзя уклониться, принятие им решений должно быть очень быстрым. Однако, решение, принятое в спешке, может оказаться неоптимальным. Рисунок 3, иллюстрирующий затраты, связанные с принятыми решениями, показывает необходимость иметь при разработке продукта методологию мониторинга рисков в режиме реального времени.

Кроме того, в «Руководстве НАСА по управлению рисками» 2011, подтверждается тот факт, что факторы риска каждого подпроцесса должны приниматься во внимание и оцениваться в начале проекта. Они окажут значительное влияние на конечную результативность (время, стоимость, качество) всего цикла разработки.

Когда входные данные для системы оценки рисков или решений оцениваются экспертами или лицами, принимающими решения, могут возникать предубеждения. В статье [2] указано: «Предполагается, что руководители принимают решение на основе данных. Но согласно многочисленным отчетам, руководители принимают решения на основе интуиции и персонального опыта.» Если входные данные необъективны, они могут серьезно снизить качество модели и

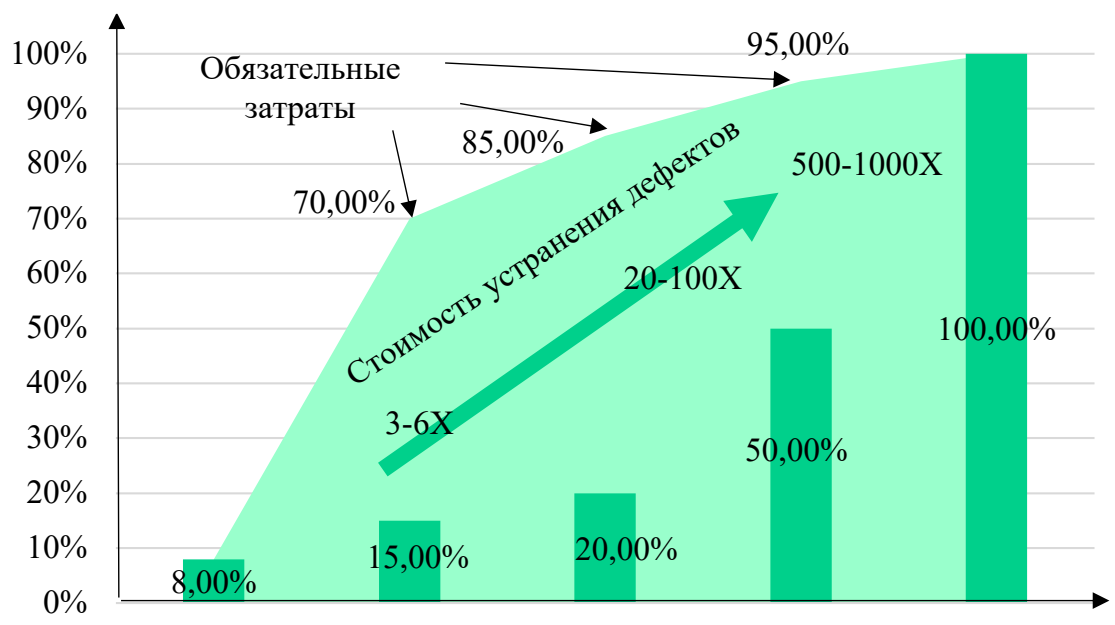

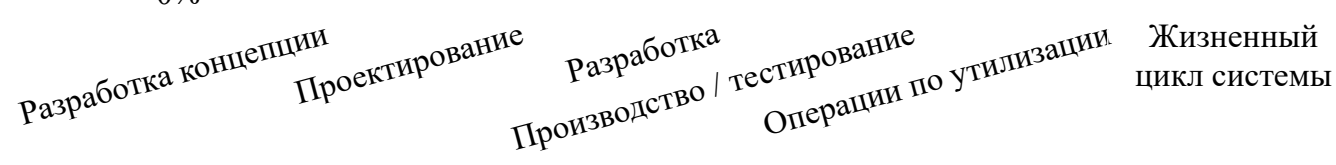

Puc. 3. Стоимость изменений в зависимости от этапов разработки [4] 
итогового анализа. В статье [11] показано, как когнитивные или мотивационные предубеждения влияют на анализ рисков; представлена классификация предубеждений в соответствии с их влиянием и трудностями, которые необходимо уменьшить. Таким образом, для повышения надежности информации, на которой основываются решения, рекомендуется, чтобы она поступала от выборки сотрудников, представляющих все уровни управления.

\section{Заключение}

Для любого инженерного проекта важно выполнение и своевременное удовлетворение требований заказчика с заложенной в бюджет стоимостью. В ходе инженерно-конструкторской деятельности риск включает несвоевременные изменения конструкции, дефекты продукции, изменчивость производства, структурные и технические сбои (материалы, инструменты, процедуры обработки, спецификации объекта), человеческий фактор и т. д. В начале, а также на протяжении всего проекта существуют подобные неопределенности, для каждой фазы разработки. Принятие правильных решений снижает риски и негативные последствия, возникающие из-за неопределенности, и, тем самым, максимизирует вероятность того, что результаты процесса разработки продукции будут соответствовать целевым значениям или даже превосходить их.

\section{Библиографический список}

1. Афанасьева, Е. А. Совершенствование процесса управления рисками, при разработке продукции космического машиностроения, путем его непрерывной интеграции // Проблемы анализа риска. - 2021. - Том 18, № 6.

2. Витчак, Е. Л., Грушицын, А.С., Данилина, М. В., Терновсков, В. Б. Проблема анализа рисков при принятии решений в бизнесе // Проблемы анализа риска. - 2020. - Том 17, № 3. - С. 74-81.

3. Chiesa, V., Frattini, F., Lazzarotti, V., \& Manzini, R. Designing a performance measurement system for the research activities: A reference framework and an empirical study //Journal of engineering and technology management. 2008. - Том 25, №. 3. - C. 213-226.

4. Gürtler, M. R., Kain A., Lindemann U. Bridging the gap: From open innovation to an open product-life-cycle by using open-x methodologies //ICoRD’13. - Springer, India. - 2013. - C. 1331-1343.

5. Impact of Technology Readiness Levels on Aerospace R\&D [Электронный ресурс]. - Режим доступа: http:// qedfusion.org/MEETINGS/0809/Weaver.pdf (дата обращения 20.06.2021)

6. Improving capability and performance with CMMI V2.0 - What has changed? [Электронный ресурс] / The Process Group. - Режим доступа: http://processgroup.com/improving-capability-and-performance-with-cmmiv2-0-what-has-changed/

7. Johansson, C., Hicks, B., Larsson, A. C., Bertoni, M. Knowledge maturity as a means to support decision making during product-service systems development projects in the aerospace sector //Project Management Journal. 2011. - Toм 42, №. 2. - C. 32-50.

8. Marle F., Pointurier C., Jaber H. Managing a complex project using a risk-risk multiple domain matrix // The Journal of Modern Project Management - 2015. Tom. 3. - C. 319-328.

9. Martínez C., Farris J. A., Letens G. Improving product development through front-loading and enhanced iteration management //IIE Annual Conference. Proceedings. - Institute of Industrial and Systems Engineers (IISE), 2011. C. 1 .

10. Mascarenhas Hornos da Costa, J., Oehmen, J., Rebentisch, E., Nightingale, D. Toward a better comprehension of Lean metrics for research and product development management //R\&D Management. - 2014. — Toм 44, №. 4. -C. 370-383.

11. Montibeller G., Von Winterfeldt D. Cognitive and motivational biases in decision and risk analysis //Risk analysis. 2015. - Tom 35, №. 7. - C. 1230-1251.

12. Pakdil F., Leonard K. M. Criteria for a lean organisation: development of a lean assessment tool //International Journal of Production Research. - 2014. - Tom 52, №. 15. - C. 4587-4607.

13. Setti, S. Project and process integration: how to usefully combine two work management models. PMI Global Congress 2010-EMEA, Milan, Italy. Newtown Square, PA: Project Management Institute. - 2010.

14. Verworn B. A structural equation model of the impact of the "fuzzy front end" on the success of new product development //Research Policy. - 2009. - Toм 38, №. 10. - C. 1571-1581.

15. What is risk maturity and how can it be sustained? [Электронный ресурс] / Risk Doctor. - Режим доступа: https:// youtu.be/zPQji2q9E9o 